\title{
Experiment Study on the Interior Sound Field of Water Filled Pipe with Elastic Wall
}

\author{
Lu Xuesong ${ }^{1, ~ a ~}$, Li Qi ${ }^{1, b}$, Liu Juan ${ }^{1, c}$ \\ ${ }^{1}$ Acoustic Science and Technology Laboratory, Harbin Engineering University, Harbin, China \\ 150001; \\ aluxuesong@hrbeu.edu.cn, bleechi@hrbeu.edu.cn, c1223953007@qq.com
}

Keywords: Elastic tube wall, water filled pipe, sound field

Abstract. This paper researched on the water filled pipe with elastic wall which is widely used in daily life. Considering the existing of the P-wave and S-wave in the pipe wall, the thin shell model and liquid wall model are not applicable. In this paper, the sound field in the water filled pipe with elastic wall will be analyzed and solved. At the same time, the experiment study on the sound field will be taken, and the insertion loss of different kinds of pipe will be got.

\section{Introduction}

The fluid filled pipe is widely used in petrochemical industry, ship and aerocraft industry, and others. In ship industry, the water filled pipe system, for example the water for life pipeline and the cooling water pipeline, is common used in every kind of ships. So, the research of water filled pipeline has excited interest of the researchers at earlier. The acoustic transmission loss through the air pipeline was studied by J. Y. Chung in 1980[1]. Lamb researched the influence of the elastic wall for the sound spread in the fluid in the pipe[2]. Fuller and Fahy discussed the wave propagation in water filled pipe and energy distribution in the water and the wall[3]. Considering the influence of the Poisson ratio, Thomson calculated the energy distribution in the wall and water[4].

This paper will solve the sound field equations of the water filled pipe with elastic wall, in order to analysis the characteristics of the acoustic propagation in the pipe. At the same time, the experiment of different kinds of pipe will be taken, and the insertion loss of different kinds of pipe will be got.

\section{The sound field in the pipe}

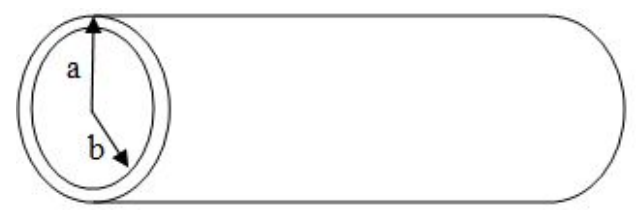

Fig 1. The model of the pipe

Suppose that the inner radius of the pipe is $b$, the outer radius is $a$, the density of the liquid in the tube is $\rho_{y}$, the sound velocity is $c_{y}$, the density of the pipe wall is $\rho_{s}, \mathrm{~S}$-wave velocity is $C_{s}$, P-wave velocity is $C_{l}$, outside the pipe is the free boundary. The velocity potential function of the pipe wall and the sound field in the pipe should satisfy the Helmholz equation:

$$
\left\{\begin{array}{l}
\nabla^{2} \Phi_{y}(r, \varphi, z)+k_{y}^{2} \Phi_{y}(r, \varphi, z)=0,0 \leq r<b \\
\nabla^{2} \Phi_{l}(r, \varphi, z)+k_{l}^{2} \Phi_{l}(r, \varphi, z)=0, b \leq r \leq a \\
\nabla^{2} \overrightarrow{\Psi_{s}}(r, \varphi, z)+k_{s}^{2} \overrightarrow{\Psi_{s}}(r, \varphi, z)=0, b \leq r \leq a
\end{array}\right.
$$


Where, $\Phi_{y} 、 \Phi_{l} 、 \vec{\Psi}$ respectively are velocity potential function of the liquid sound filed in the tube velocity potential function of P-wave in the tube wall and velocity potential function of S-wave in the tube wall, $k_{y}=\frac{\omega}{c_{y}}, k_{l}=\frac{\omega}{c_{l}}, k_{s}=\frac{\omega}{c_{s}}$ 。The general solution of (1) is:

$$
\left\{\begin{array}{l}
\Phi_{y}(r, \varphi, z, t)=\sum_{m=0}^{\infty} A_{m} J_{m}\left(\xi_{y} r\right)\left[\begin{array}{l}
\cos (m \varphi) \\
\sin (m \varphi)
\end{array}\right] \exp \left[j\left(k_{z} z-\omega t\right)\right] \\
\Phi_{l}(r, \varphi, z, t)=\sum_{m=0}^{\infty} B_{m} P_{m}\left[\begin{array}{l}
\cos (m \varphi) \\
\sin (m \varphi)
\end{array}\right] \exp \left[j\left(k_{z} z-\omega t\right)\right] \\
\psi_{s}(r, \varphi, z, t)=\sum_{m} Q_{m}\left[\begin{array}{l}
\cos (m \varphi) \\
\sin (m \varphi)
\end{array}\right] \exp \left[j\left(k_{z} z-\omega t\right)\right]
\end{array}\right.
$$

Where, $P_{m}=J_{m}\left(\xi_{l} a\right) N_{m}\left(\xi_{l} r\right)-J_{m}\left(\xi_{l} r\right) N_{m}\left(\xi_{l} a\right), Q_{m}=J_{m}\left(\xi_{s} a\right) N_{m}\left(\xi_{s} r\right)-J_{m}\left(\xi_{s} r\right) N_{m}\left(\xi_{s} a\right)$, $k_{z}^{2}+\xi_{0}^{2}=k_{0}^{2}, \quad k_{z}^{2}+\xi_{l}^{2}=k_{l}^{2}, \quad k_{z}^{2}+\xi_{s}^{2}=k_{s}^{2}$.Proved $\vec{\Psi}=C_{m} \vec{X}+D_{m} \vec{Y}$ is satisfied with (1), where $\vec{X}=\nabla \times \psi_{s} \overrightarrow{e_{z}}, \quad \vec{Y}=\nabla \times \vec{M}$. So there is:

$$
\vec{\Psi}=\sum_{m=0}^{\infty}\left\{\begin{array}{l}
{\left[\begin{array}{l}
\left.C_{m} \frac{m}{r} Q_{m}\left[\begin{array}{l}
-\sin (m \varphi) \\
\cos (m \varphi)
\end{array}\right]+D_{m} j k_{z} Q_{m}^{\prime}\left[\begin{array}{l}
\cos (m \varphi) \\
\sin (m \varphi)
\end{array}\right]\right] e^{j\left(k_{z} z-\omega t\right)} \overrightarrow{e_{r}} \\
\left.-C_{m} Q_{m}^{\prime}\left[\begin{array}{l}
\cos (m \varphi) \\
\sin (m \varphi)
\end{array}\right]+D_{m} j k_{z} \frac{m}{r} Q_{m}\left[\begin{array}{l}
-\sin (m \varphi) \\
\cos (m \varphi)
\end{array}\right]\right] e^{j\left(k_{z} z-\omega t\right)} \overrightarrow{e_{\varphi}}
\end{array}\right.} \\
+D_{m}\left(\frac{m^{2}}{r^{2}} Q_{m}-Q_{m}^{\prime \prime}\right)\left[\begin{array}{l}
\cos (m \varphi) \\
\sin (m \varphi)
\end{array}\right] e^{j\left(k_{z} z-\omega t\right)} \overrightarrow{e_{z}}
\end{array}\right\}
$$

Whereby, we can obtain the total sound velocity in the elastic wall, which is :

$$
\begin{aligned}
& \vec{v}=\nabla \Phi+\nabla \times \vec{\Psi}
\end{aligned}
$$

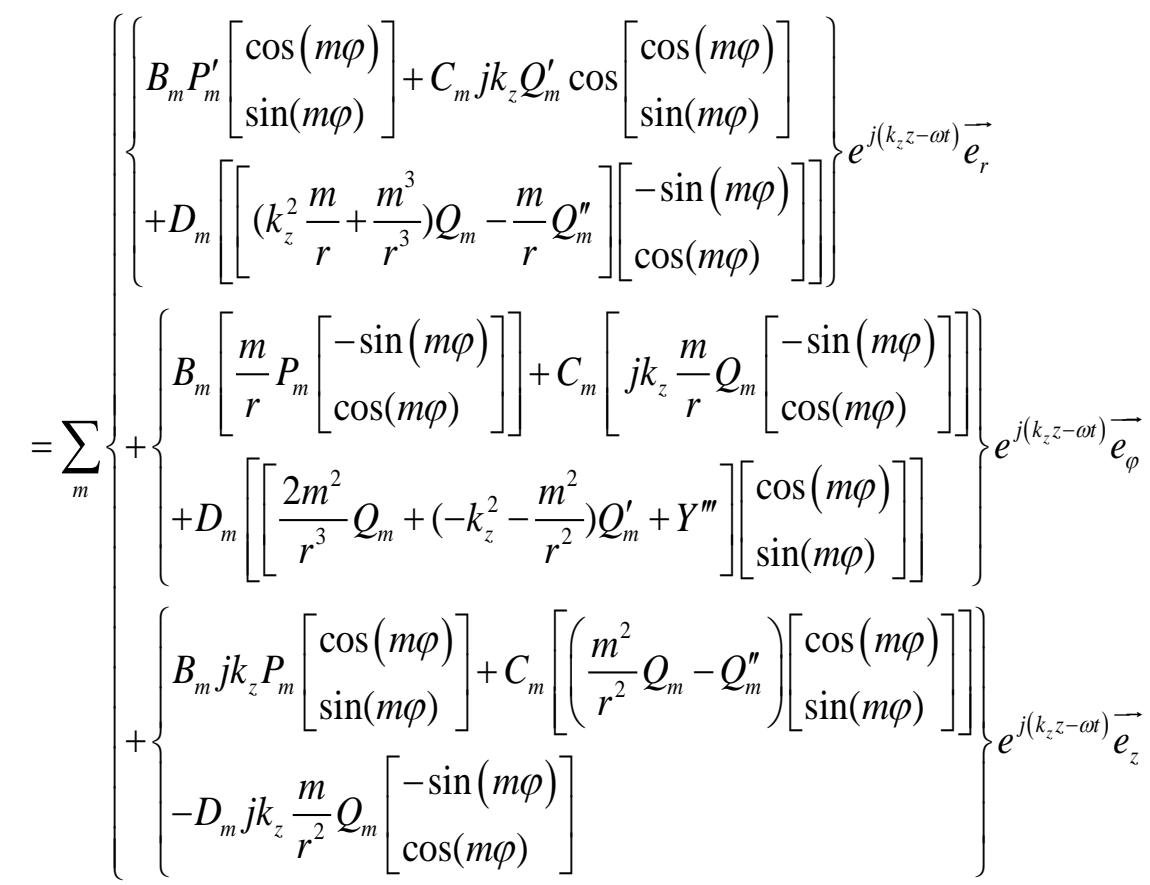

The boundary conditions can be expressed as:

(1) When $r=b$, particle velocity is continuous in the vertical direction: 


$$
-\left.\frac{\partial \Phi_{y}(r, \varphi, z)}{\partial r}\right|_{r=b}=\left.\overrightarrow{v_{r}}\right|_{r=b}
$$

(2) When $r=b$, sound pressure is continous in the vertical direction between liquid medium and elastic medium:

$$
\left.P_{0}\right|_{r=b}=\left.T_{r r}\right|_{r=b}
$$

(3) When $r=a$, tangential stress $T_{r \varphi}$ in elastic medium is zero:

$$
\left.T_{r \varphi}\right|_{r=a}=\left.\mu\left(\frac{\partial v_{\varphi}}{\partial r}+\frac{1}{r} \frac{\partial v_{r}}{\partial \varphi}-\frac{v_{\varphi}}{r}\right)\right|_{r=a}=0
$$

(4) When $r=a$, tangential stress $T_{r z}$ in elastic medium is zero:

$$
\left.T_{r z}\right|_{r=a}=\left.\mu\left(\frac{\partial v_{r}}{\partial z}+\frac{\partial v_{z}}{\partial r}\right)\right|_{r=a}=0
$$

So we can obtain the eigenvalue equation and intrinsic value of the sound field in the tube. Whereby, the Cut-off frequency of the sound field in the elastic tube is can be obtained. The acoustic wave of which frequency is below the cut-off frequency can not propagate in the pipe.

\section{Experimental study of the sound field of the water filled pipe with elastic wall}

Select two different material pipes, one is the steel pipe, of which the outer diameter is $6 \mathrm{~cm}$, the inner diameter is $3 \mathrm{~cm}$, and the tube length is $140 \mathrm{~cm}$, another material is the PPR (pentatrico-peptide-repeats),of which the outer diameter is $11.4 \mathrm{~cm}$, the inner diameter is $9.8 \mathrm{~cm}$,and the tube length is $200 \mathrm{~cm}$. During the experiment the transmitting transducer and the receiving transducer respectively are 8105 hydrophone and 8103 hydrophone of B \& K company.

Keep the transmitting transducer enclosed in one end of pipe, and connect the other end of the pipe with the reverb tank. Then use the reverberation method to measure the sound radiation power of the sound waves radiated by transmitting transducer after which go through the pipe. When the pipe directly is rigidly connected to the reverb tank, the sound radiation power of the sound waves which is obtained in the reverb tank is as follows:

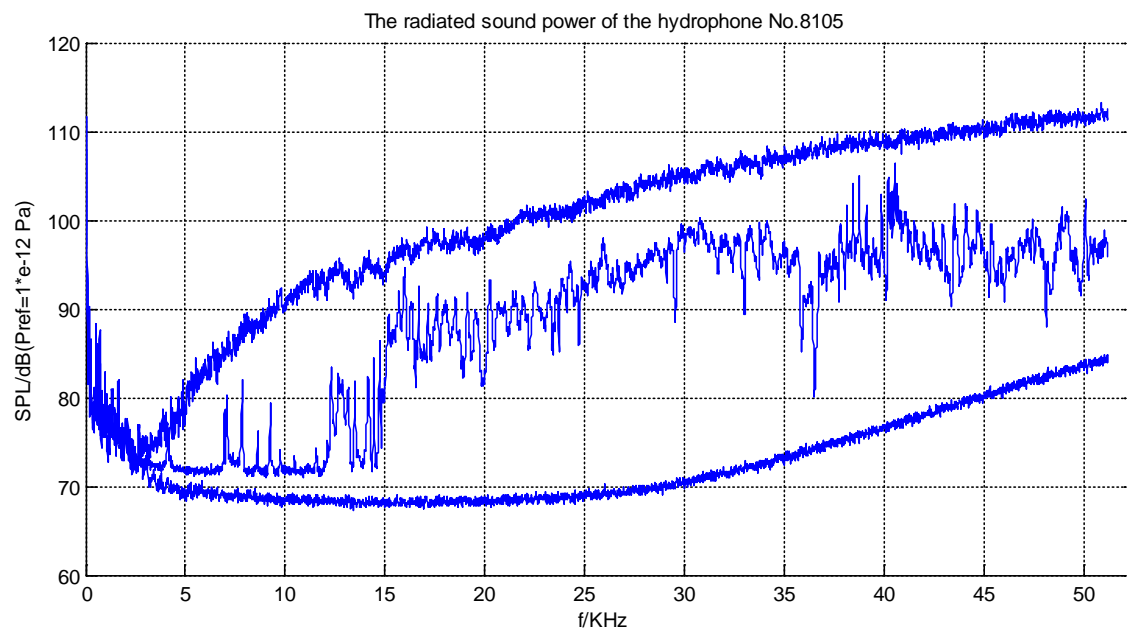

Fig 2. transducer going through the pipe contrast with the sound radiation power of the sound waves in the reverb tank

The upper curve in Fig 2 is the sound radiation power of the transducer measured in reverb tank, the following curve is the background noise, and the middle curve is the sound radiation power of the sound waves which go through the pipe. You can find several line spectrum signal exists between $5 \mathrm{KHz}-15 \mathrm{KHz}$.As of now, These signal is resonance signals of the pipeline motivated by the transducer. Owing to the tube whose diameter is smaller than its length, and tube wall is thick, we can 
use beam model to analyze. So we can get a conclusion: When one end of the pipe is fixed, the other is free, the vibration frequencies of the beam is shown in equation[3]:

$$
f_{0}=\frac{c}{4 l}(2 n-1),(n=1,2,3 \cdots)
$$

In a word, the resonant frequency of the steel pipe can be calculated. Through contrasting with the frequency of the line spectrum signal, we can get the table 1:

Table 1: The resonant frequency of the steel pipe and the measured

\begin{tabular}{|c|c|c|c|c|c|c|}
\hline $\mathrm{n}$ & 9 & 15 & 16 & 18 & 19 & 20 \\
\hline $\begin{array}{c}\text { The resonant frequency } \\
\text { (theoretical value) }\end{array}$ & 4308.21 & 7349.31 & 7856.16 & 8869.86 & 9376.71 & 9883.56 \\
\hline $\begin{array}{c}\text { The resonant frequency } \\
\text { (measurements value) }\end{array}$ & 4112 & 7080 & 7840 & 8616 & 9248 & 9752 \\
\hline $\mathrm{n}$ & 23 & 25 & 26 & 27 & 28 & 29 \\
\hline $\begin{array}{c}\text { The resonant frequency } \\
\text { (theoretical value) }\end{array}$ & 11404.1 & 12417.8 & 12924.6 & 13431.5 & 13938.4 & 14445.2 \\
\hline $\begin{array}{c}\text { The resonant frequency } \\
\text { (measurements value) }\end{array}$ & 11550 & 12300 & 12810 & 13480 & 14130 & 14420 \\
\hline
\end{tabular}

You can find the line spectrum signal is the resonant frequency of the steel pipe.

In order to prevent vibration of the steel pipe from affecting the outcome of sound field measurements in the reverb tank, paste the steel pipe to the reverb tank through soft rubber. Equivalent to add a short-soft- connected devices between steel pipe and reverb tank. Re-measured, we can get the result as flows in the figure 3.

By comparison of figure 2 and 3, That line spectrum signal is caused by resonance of the steel pipe is once again proved. In addition, it can be seen that the steel pipe has a good effect on cutting off the sound waves whose frequency is below $15 \mathrm{kHz}$ in Figure 3.

Using the PPR to re-measure, the results shown in the figure 4.

Can be found the cut-off frequency of sound waves constantly moves to lower frequency, with the increase of the inner diameter of the pipe.

Meanwhile, due to the different sizes of the different pipes, the sound field of the pipe has different effect on sound source, Which led to different radiation impedance, when the sound source is in the different pipes. so the sound field of radiation effects after the cut-off frequency is also different.

According to figure 3 and figure 4, we may also obtain the insertion loss of the different pipes in figure 5 and 6 .

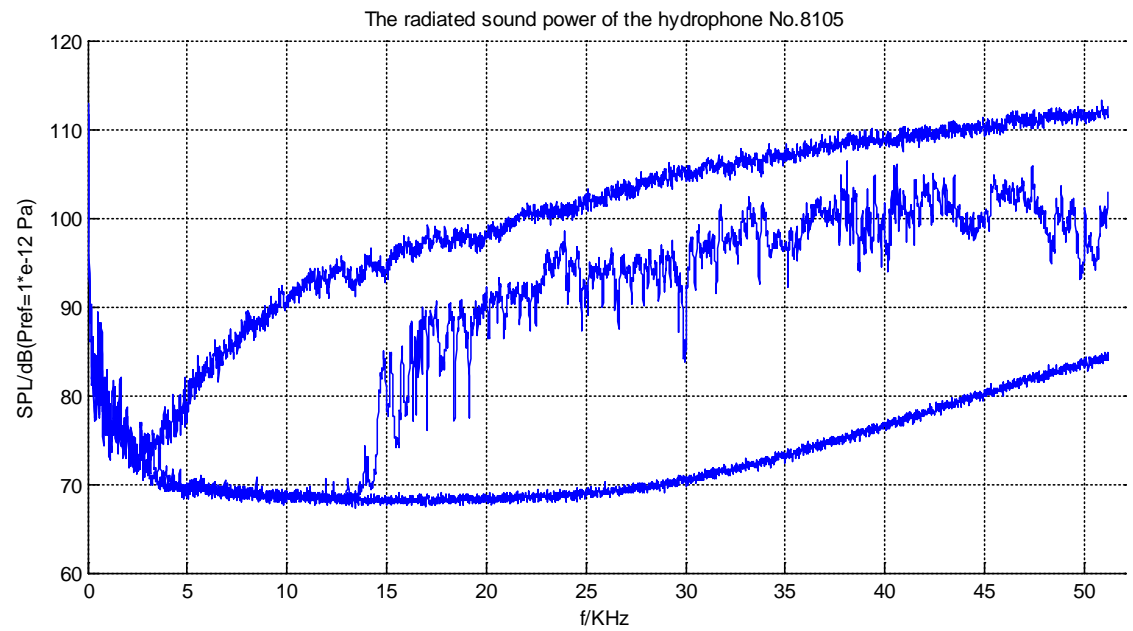

Fig 3. transducer going through the pipe contrast with the sound radiation power of the sound waves in the reverb tank. 


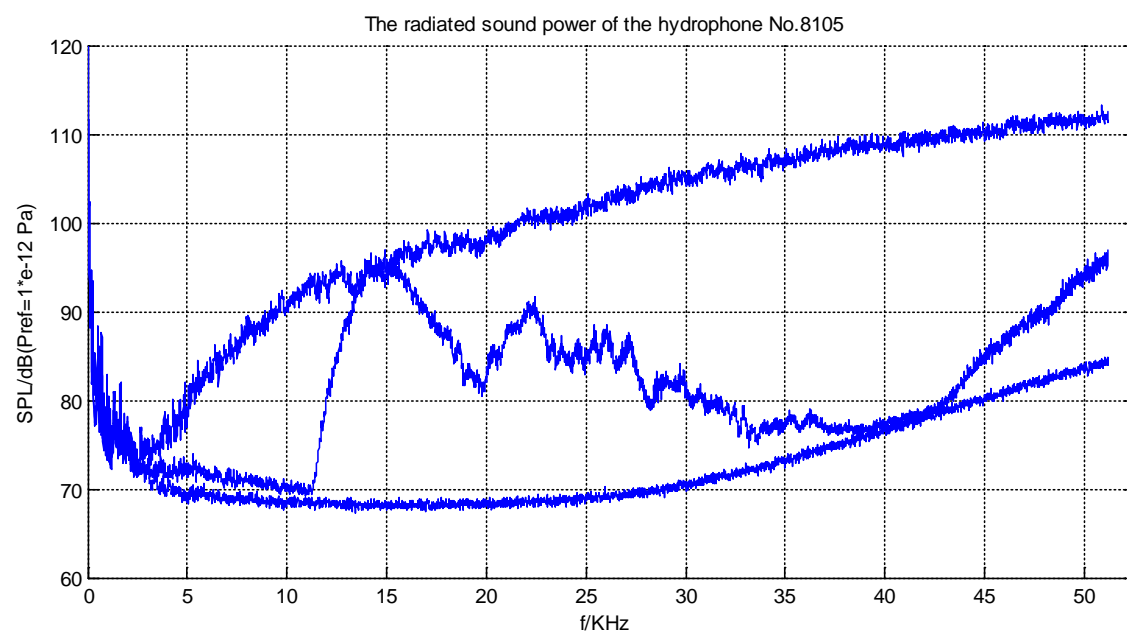

Fig 4. transducer going through the pipe contrast with the sound radiation power of the sound waves in the reverb tank.

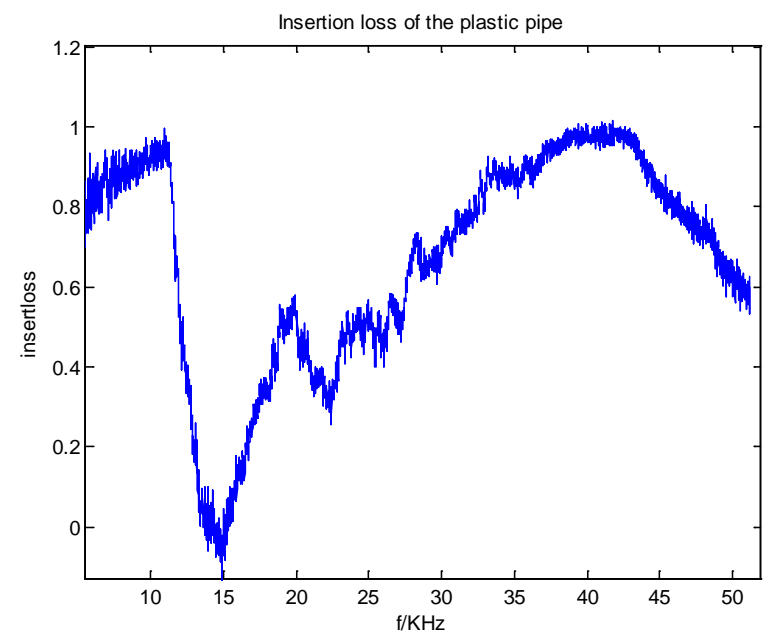

Fig 5. Insertion loss of the plastic pipe

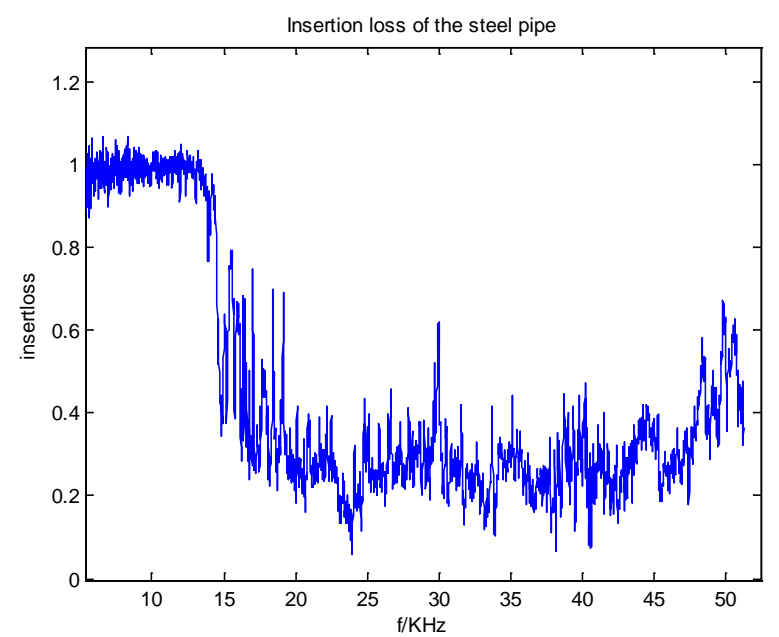

Fig 6. Insertion loss of the steel pipe

\section{Conclusion}

Due to the different structure and parameters of the pipe, the cut-off frequency of sound waves is not the same in the different pipe. At the same time, the same sound source has different radiation in the pipes, owing to the sound filed in the pipe having effect on the surface of the sound source. This paper gives insertion loss of two different pipe. The sound field in the pipe influences the radiation of the sound source, which is the focus of the next work.

\section{References}

[1] J. Y. Chung and D. A. Maser. Transfer function method of measuring in-duct acoustic properties[J]. Journal of the Acoustical Society of America. Vol. 68 -3(1980),p. 907.

[2] Lamb, A., On the velocity of sound in a tube, as affected by the elasticity of the walls,. Manchester Literary and philosophical Society-Memoirs and Proceedings, 1898,42(9).

[3] Fuller, Fahy. Characteristics of wave propagation and energy distributions in cylindrical elastic shells filled with fluid. J. Sound Vib., 1982; 81:501-518

[4] W. T. Thomson. Transmission of pressure waves in liquid filled tubes, Proceeding of the first U.S. National Congress on Applied Mechanics, Chicago, 1953:922-933

[5] H. L. Zhang. Theoretical Acoustics. 2012; 119-131. 